\title{
Cardiac induced localised motion of the human torso detected by a long period grating fibre optic sensing scheme
}

\author{
T. Allsop*, G. Lloyd ${ }^{2}$, R. S. Bhamber ${ }^{3}$, L. Hadzievski ${ }^{4}$, M. Halliday ${ }^{5}$, D. J. Webb \\ Authors' Affiliations \\ Aston Institute of Photonic Technologies, Aston University, Birmingham, B4 7ET, U.K \\ ${ }^{2}$ Moog Insensys Ltd, Ocean House, Whittle Avenue, Segensworth West, Fareham, PO15 \\ 5SX, U.K \\ ${ }^{3}$ Instituto de Óptica "Daza de Valdés", (C.S.I.C.), C/ Serrano, 121, 28006, Madrid, Spain \\ ${ }^{4}$ Vinča Institute of Nuclear Sciences, University of Belgrade, P.O. Box 522, 11001 \\ Belgrade, Serbia \\ ${ }^{5}$ Cytec Process Materials (Keighley) Ltd, 500 Bradford Road, Keighley West Yorkshire. \\ BD20 5NG, U.K.
}

\begin{abstract}
Cardiovascular health of the human population is a major concern for medical clinicians, with cardiovascular diseases responsible for $48 \%$ of all deaths worldwide, according to the World Health Organisation. Therefore the development of new practicable and economical diagnostic tools to scrutinise the cardiovascular health of humans is a major driver for clinicians. We offer a new technique to obtain seismocardiographic signals covering both ballistocardiography (below $20 \mathrm{~Hz})$ and audible heart sounds (20Hz upwards). The detection scheme is based upon an array of curvature/displacement sensors using fibre optic long period gratings interrogated using a variation of the derivative spectroscopy interrogation technique
\end{abstract}

\section{Introduction}

Shape sensing and structural health monitoring ${ }^{1,2}$ have been a prominent motivation for the research and development of fibre optical sensors in the past, and continue to be so. Over approximately the last 15 years there has been a rapid increase in both the types of fibre optical sensors and the range of applications ${ }^{3,4}$, with increasing interest in medical applications ${ }^{5,6}$. Cardiorespiratory measurement was an early application of these types of sensors and continues to be an active field of research ${ }^{7,8}$. The main interest in these sensors is related to their potential low cost of production, ease of handling, potential for ambulatory application, small size and relatively low skill requirements for operation. Until recently the most popular sensing element used was the fibre Bragg grating ${ }^{9}$; this type of sensor has good strain and temperature response but is insensitive to other environmental parameters.

Heart rate variability has been used to investigate how the autonomous nervous system controls the heart. Differences in the heart's dynamics at frequencies up to $0.5 \mathrm{~Hz}$ relate to altered control of the cardiovascular system ${ }^{10}$. Heart malfunction can also be identified by recording and analysing the body vibrations caused by the mechanical activity of the heart. Measurements of the ballistic forces on the heart are made at higher frequencies. These forces occur on the sudden ejection of blood into the great vessels with each heartbeat. The predominant frequency range associated with this technique is 1 to $40 \mathrm{~Hz}$ and detection can be made non-invasively at the chest surface ${ }^{11,12}$. It is also known that the sounds or mechanical motions of a single cardiac cycle of a human heart can be divided into two dominant events ${ }^{13}$. The first event is associated with the onset of systole, and is produced by the closure of the mitral and tricuspid valves producing frequencies up to $200 \mathrm{~Hz}^{14}$. The second set of mechanical sounds is produced by the closure of the aortic and pulmonary valves, and it denotes the end of systole and the beginning of diastole with a frequency range of $500 \mathrm{~Hz}^{15,16}$. Researchers have used a multimode graded index optical fibre clamped between a pair of micro-benders and exploited the variations in intensity to detect the ballistic forces of the heart ${ }^{17}$. This shows promise but additional bends in the fibre may occur during the measurement procedure which can be misinterpreted or generally corrupt the data from the sensor and furthermore the procedure provides a large regional measurement.

An optical fibre long period grating curvature sensing scheme with a response time of $54 \mathrm{~Hz}$ is presented in this paper that has sufficient sensitivity to detect the mechanical motions or sounds of the human heart non-invasively from a subject's body surface. We detect peak harmonics at frequencies of $1.3 \mathrm{~Hz}, 7.6 \mathrm{~Hz}, 10.8 \mathrm{~Hz}$ and $22 \mathrm{~Hz}$ in healthy human subjects. These results are consistent with other published results ${ }^{18}$. In the past similar data have been used to identify malfunctioning hearts and the results in this paper suggest that our scheme may offer a low-cost aid in the detection of heart problems, though detailed clinical trials would of course be required.

23rd International Conference on Optical Fibre Sensors, edited by José Miguel López-Higuera,

Julian Jones, Manuel López-Amo, José Luis Santos, Proc. of SPIE Vol. 9157, 91574N

(C) 2014 SPIE - CCC code: 0277-786X/14/\$18 - doi: 10.1117/12.2059089 


\section{Sensors and Sensing Scheme}

A series of long period gratings (LPGs) was fabricated in progressive three layered (PTL) fibre (supplied by Fibercore Ltd) as the sensing elements. These sensors ${ }^{19}$, were fusion spliced into a line of standard single mode telecommunications fibre; typical physical parameters of the PTL fibre can be found elsewhere ${ }^{19}$. The periods of the LPGs were chosen to spectrally match the distributed feedback (DFB) lasers used to monitor the response of the LPG sensors within a specific curvature range. The interrogation scheme is an adaptation of the derivative amplitude spectroscopy approach ${ }^{20}$, in which a pair of spectrally separated DFB lasers is used to monitor the gradients of the spectral feature of the sensor over a specific spectral bandwidth, with the ratio of these two signals being used to recover the curvature of sensors. In this case the fibre pigtailed DFB laser modules are emitting at wavelengths of $1470.46 \mathrm{~nm}$ and $1470.73 \mathrm{~nm}$; a spectral separation of $0.27 \mathrm{~nm}$. The sensors are interrogated by switching between the DFB lasers, with synchronous detection for both lasers. Each laser is swept over a small wavelength range and then the ratio of these two signals is used as the sensor's response to curvature, which stops bending induced loss elsewhere in the system affecting the performance.

A typical spectral transmission response to curvature obtained with an optical spectrum analyser is shown in figure 1, after the sensing elements and some of the optical fibre had been encapsulated into a synthetic low curing $\left(90^{\circ} \mathrm{C}\right)$ silicone rubber. The spectral sensitivity of the sensors with respect to curvature; $\Delta \lambda / \Delta R$ varied from $2.9 \mathrm{~nm} \mathrm{~m}$ to $8.9 \mathrm{~nm}$ m over the range of curvature but the DBF laser used for interrogation has a wavelength of $1470 \mathrm{~nm}$ which leads to a spectral sensitivity of $\Delta \lambda / \Delta R \approx 6.80 \mathrm{~nm} \mathrm{~m}$. The spectral response to curvature can be considered reasonably linear over a small range of curvature ${ }^{8}$. The complete spectral wavelength response of the curvature sensor can be represented by a $4^{\text {th }}$ order polynomial, resulting in a curvature error (root mean square deviation from the polynomial) of $\pm 0.01 \mathrm{~m}^{-1}$.

Implementing the derivative spectroscopy interrogation technique ${ }^{8}$ introduces an error of \pm 0.01 . This error was obtained by fitting a $6^{\text {th }}$ order polynomial to the ratio of the derivatives obtained from the two lasers, which is used to calibrate the scheme for curvature. This error associated with the derivatives can be translated into a curvature error of $\pm 0.014 \mathrm{~m}^{-1}$ for the sensing scheme. This is a comparable resolution to that reported elsewhere with a similar interrogation scheme $^{8}$. The data acquisition rate was $54 \mathrm{~Hz}$. A schematic of the entire sensing scheme is shown in figure 1a. The transmission spectra of the LPG that are shown in figure 1a are obtained using light from a broadband light source and monitored using an optical spectrum analyser. The curvature measurements of the LPGs that are shown in figure $1 \mathrm{~b}$ were obtained using a conventional two point bending test procedure with the fibre LPGs laid upon a thin stainless steel strip (thickness of $3.04800 \times 10^{-3} \mathrm{~m}$ ). The laser modules used in the interrogator typically had optical powers of $5 \mathrm{~mW}$ and nominal bandwidths of $20 \mathrm{MHz}$ and was cooled thermo-electrically. The interrogator was controlled using bespoke software via a USB connection from a Laptop (dual-core).
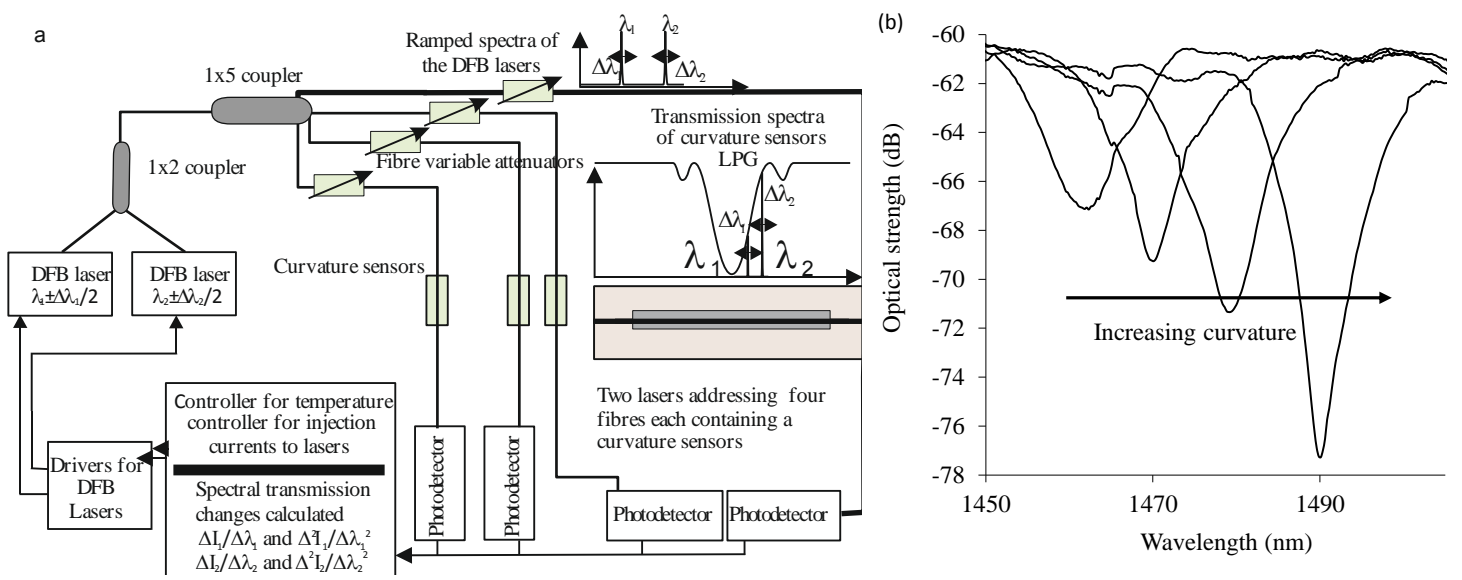
(be interrogation/multiplexing scheme for detecting the mechanical movement of the human heart. (b) Shows the variation in the transmission spectrum of the sensor as a function of curvature. The LPG sensing element has a period of $540 \mu \mathrm{m}$ and a length of $5 \mathrm{~cm}$.

\section{Detection of mechanical motion of the human heart}

A series of experiments was conducted with varying data acquisition rates and subject postures. Firstly, six locations on the human torso were used to monitor the curvature variation caused by the mechanical motion of the heart. To reduce signal corruption due to torso respiratory movement, the subjects held their breath for 10 seconds, though the authors acknowledge that this will affect the mechanical behaviour of the heart. This experiment was to confirm that the 
mechanical motion of the heart can be detected from various locations on the torso. Results were obtained using the same curvature sensor at each location so a reasonable comparison can be made between locations, though it is noted that the mean curvature is different at the various placements which yields slightly different sensitivities. The sensor locations on the subject's torso are shown schematically in figure 2a and some examples of the detected curvature variation created by the mechanical motion of the heart are shown in figures $2 \mathrm{~b}$ to $2 \mathrm{~g}$, all records being obtained with the same posture (neutral spine position).
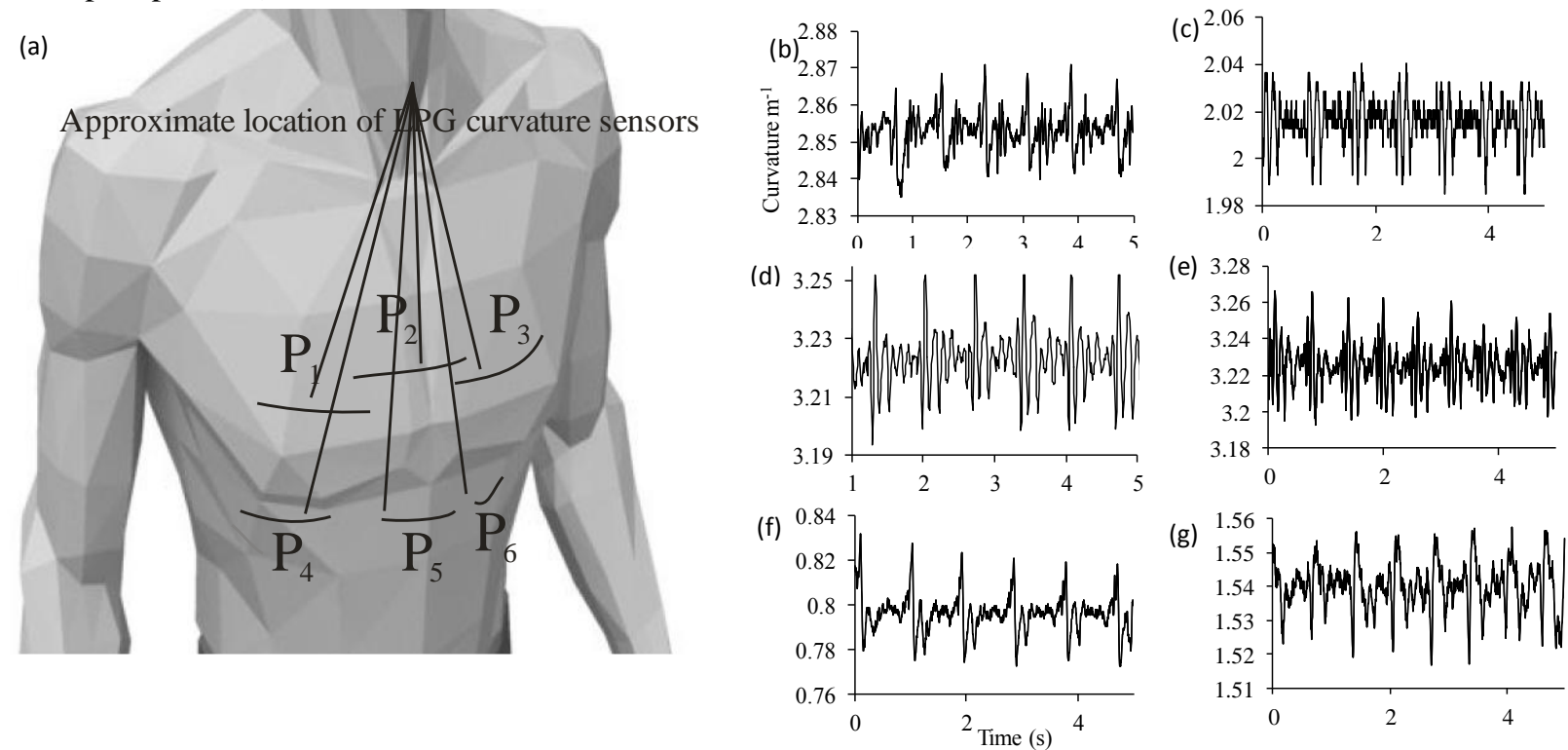

Figure 2 (a) Schematic of sensor locations on the human torso. The plots show the temporal curvature variation of the sensors associated with mechanical motion of the human heart. The sensors are adhered to the surface of the human torso at various locations (b) position $\mathrm{P}_{1}$, (c) position $\mathrm{P}_{2}$, (d) position $\mathrm{P}_{3}$, (e) position $\mathrm{P}_{4}$, (f) position $\mathrm{P}_{5}$ and (g) position $\mathrm{P}_{6}$.

On inspection, figures $2 \mathrm{~b}$ to $2 \mathrm{~g}$ show there are different waveforms for each sensing location, obtained with a data acquisition rate of $54 \mathrm{~Hz}$. This is as expected given the different biological materials through which the mechanical wave is propagating and asymmetry in the heart's motion. Performing Fourier transforms on the collected data produce similar harmonics at each spatial location, see figure 3 . The harmonics are $1.28 \mathrm{~Hz} \pm 0.26 \mathrm{~Hz}, 7.6 \mathrm{~Hz} \pm 1.1 \mathrm{~Hz}, 10.8 \mathrm{~Hz} \pm 1.5 \mathrm{~Hz}$ and $22.1 \mathrm{~Hz} \pm 1.1 \mathrm{~Hz}$ and the error is the standard deviation of the variation of the harmonics.

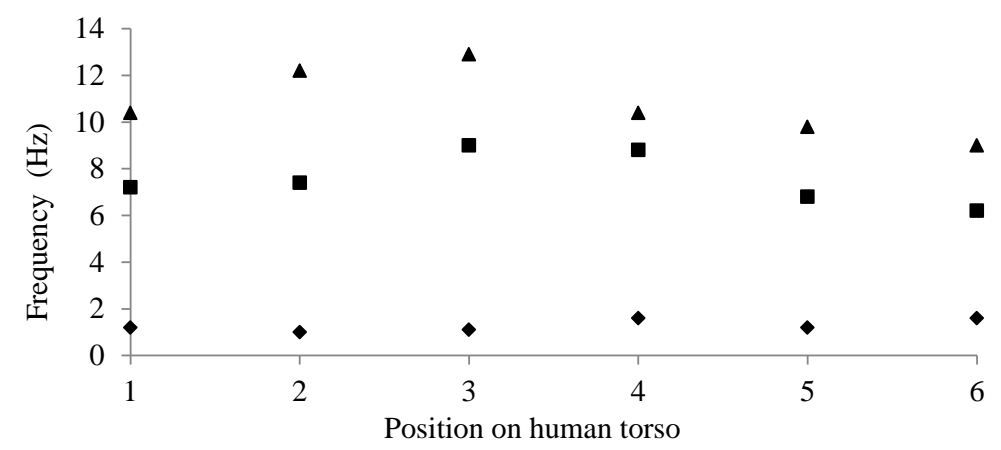

Figure 3 The detected harmonics from the waveform generated from the mechanical motion of the human heart at six spatial locations.

Comparing the scheme demonstrated here to other methods reported in the literature shows this one to have several advantages. Firstly, the hardware to implement this scheme is small, lightweight, has ease of maintenance and the potential to be inexpensive compared to the classical procedure used for ballistocardiography (BCG) that is bulky, expensive, requires significant mechanical maintenance, and is cumbersome to operate ${ }^{21}$. Secondly, researchers have recently addressed the awkwardness of operation of the traditional approach by using force sensitive electromechanical films ${ }^{11,12}$ and pressure pads ${ }^{22,23}$ but these yield an overall body signal. As a consequence, signals cannot be attributed to specific regions of the torso whereas the scheme presented in this paper can make small regional measurements. Thirdly, the small regional measurement provided by our system permits the study of the mechanical behaviour of the human 
heart from various perspectives, providing the potential to recover the mechanical motion of the heart in 3-dimensons. Fourthly this scheme's sensors are inherently immune from electromagnetic interference and are chemically inert.

The author believe that this is the first time that LPG sensors have used to detect in real-time the mechanical motion of the heart at various frequencies below and above $20 \mathrm{~Hz}$. To demonstration the potential usefulness of the LPG sensors in detecting the mechanical motion of the heart and potential applications in the field of cardiology the LPG sensor was positioned at three locations across the front region of the thorax and approximately in line with the midposition of the heart and at three locations across the front upper abdominal region just below the apex of the human heart. We detected peak harmonics at frequencies of $1.3 \mathrm{~Hz}, 7.6 \mathrm{~Hz}, 10.8 \mathrm{~Hz}$ and $22.1 \mathrm{~Hz}$ in healthy human subjects. These results are consistent with other published results ${ }^{24}$.

The authors of this paper realise that these results by themselves are not sufficient to validate the scheme as a new diagnostic medical tool. Considerable medical trials and analysis will be needed to confirm whether this sensing scheme could be an effective aid in the detection of heart malfunctions. However, the authors do feel that the results show our approach to have potential in the field of cardiology.

\section{References}

[1] M. Davis, et al, "Shape and vibration mode sensing using a fiber optic Bragg grating array", Jn Smart Mater. Struct., No.5, pp.759$765,(1996)$.

[2]R. Bhamber, et al, "Arbitrary real-time three dimensional corporal object sensing and reconstruction”, Optics Letts., Vol. 37, No. 17, pp. 3549-3551, (2012) .

[3] S. Vasilev, et al, "Long-period refractive index fibre gratings: properties, applications and fabrication techniques", Proc. SPIE 4083, pp.212-223, (2000).

[4] T. Allsop, et al, "Surface Plasmon Resonance Generation Utilising Gratings for Biochemical Sensing", OFS-18 Cancun Mexico, paper WA4 , (2006).

[5] G. Wehrle, et al , "A fibre optic Bragg grating strain sensor for monitoring ventilatory movements", Meas. Sci. Technol., Vol. 12, pp.805-809, (2001).

[6] A. Leung, "A review of fiber-optic biosensors", Sensors and Actuators B, vol. 125, pp. 688-703, (2007).

[7] L-G. Lindberg, et al, "Monitoring of respiratory and heart rates using a fibre-optic sensor", Medical \& Biological Engineering \& Computing, Vol. 30, pp.533-537, (1992).

[8] T. Allsop, et al, "Respiratory function monitoring using a real-time three dimensional fibre optic shaping sensing scheme based upon fibre Bragg gratings, Jn Biomedical Optics, Vol. 17, 117001, (2012).

[9] I. Bennion, et al, "Uv-written in-fibre Bragg gratings", Optical and Quantum Electronics, Vol. 28, No. 2, pp.93-135, (1996).

[10] A. Schäfer, et al, "How accurate is pulse rate variability as an estimate of heart rate variability? A review on studies comparing photoplethysmographic technology with an electrocardiogram", International Journal of Cardiology, early Posting. (2012).

[11] J. Siivola, "New noninvasive piezoelectric transducer for recording of respiration, heart rate and body movements," Med. Biol. Eng. Comput., vol. 27, pp. 423-424, (1989).

[12] S. Junnila, et al, "An EMFi-film sensor based ballistocardiographic chair: Performance and cycle extraction method," in Proc. IEEE Workshop on Signal Process. Syst. Design and Implementation, Athens, Greece, pp. 373-377, (2005).

[13] B. Kirby, et al, "Clinical examination of the heart," Medicine, Vol. 34, pp. 123-128, 2006.

[14] R. S. Anand, R. S., "PC based monitoring of human heart sounds," Comput. Elect. Eng., Vol. 31, pp. 166-173, (2005).

[15] P. Arnott, et al, "Spectral analysis of heart sounds: Relationships between some physical characteristics and frequency spectra of first and second heart sounds in normals and hypertensives," J. Biomed. Eng., Vol. 6, pp. 121-128, (1984).

[16] A. Vander, A. Sherman, J. Luciano, D., Human Physiology: The Mechanisms of Body Function. New York: McGraw-Hill, (1990).

[17] Z. Chen, et al, "Portable fiber optic ballistocardiogram sensor for home use.", SPIE Vol. 8218, Optical Fibers and Sensors for Medical Diagnostics and Treatment Applications XII, pp. 82180X-1- 82180X-7. (2012).

[18] X. Zhu, et al, "Real-Time Monitoring of Respiration Rhythm and Pulse Rate During Sleep", IEEE Trans. On Biomedical Engineering, Vol.53, No. 12, pp.2553-2563, (2006).

[19] T. Allsop, et al," A comparison and characteristics of sensing parameters of long period gratings written in three different types of fiber", Optical Fiber Technology, Vol. 9, Issue 4, pp 210-223, (2003).

[20] T. Allsop, et al, "A strain sensing system using a novel optical fibre Bragg grating sensor and a synthetic heterodyne interrogation technique", Meas. Sci. Tech., 13 (5), pp. 731-740, (2002).

[21] O. T. Inan, et al, "Robust ballistocardiogram acquisition for home monitoring", Physiol. Meas. , 30, 169-185 (2009).

[22] D. C. Mack, et al, "Development and preliminary validation of heart rate and breathing rate detection using a passive, Ballistocardiography-based sleep monitoring system”, IEEE Trans., Inf. Technol. Biomed., 13(1): 111-120 (2009).

[23] Z. Chen, et al, "Portable Fiber Optic Ballistocardiogram Sensor for Home Use", Proc. of SPIE Vol. 8218, Optical Fibers and Sensors for Medical Diagnostics and Treatment Applications XII, pp. 82180X-1- 82180X-7, (2012).

[24] P. S. Rahko, "Evaluation of the skin-to-heart distance in the standing adult by two-dimensional echocardiography." Jn American Society of Echocardiography, Vol. 21, No. 6, pp.761-764, (2008). 